\title{
IMPLEMENTATION OF A QUALITY APPROACH IN ENGINEERING AT UQAM
}

\author{
Guy Bégin ${ }^{1}$ and Patrick Charland ${ }^{2}$ \\ ${ }^{1}$ Department of Computer Science, ${ }^{2}$ Department of Didactic \\ UQAM, P.O. Box 888, Station Centre-Ville, Montréal H3C 3P8 \\ begin.guy@uqam.ca; charland.patrick@uqam.ca
}

\begin{abstract}
For facilitating the transition to engineering attributes, engineering professors at UQAM have teamed up with colleagues in educational sciences who have developed expertise through similar endeavors in the field of teacher training. The collaboration will allow a broadening of the reflection on professional training that will benefit both parties, as well as the creation of tools, procedures and mechanisms that can be transfered to other schools and faculties.
\end{abstract}

Keywords: Quality, Engineering, Education, Assessment, Criteria, Indicators

\section{INTRODUCTION}

Like other schools and faculties of engineering across Canada, UQAM is in the process of aligning its accredited engineering program to the engineering attributes as defined by the CEAB. To make the changeover easier, engineering professors have decided to team up with colleagues in educational sciences, to benefit from the expertise developed through similar experiences in recent years, especially in the field of teacher training. Indeed, ten years ago, a committee responsible for accrediting teacher training programs in Quebec universities implemented an approach oriented on the development of professional competencies. The collaboration between engineering and education professors will allow a broadening of the reflection on professional training that will benefit both parties.

\section{PROCESS}

A number of steps have been taken in this process. Professors involved in the microelectronics engineering program have identified, for each of their courses, where, when and how the engineering attributes are developed and evaluated. Based on the information gathered, a detailed mapping of the attributes in the curriculum is currently being completed.

In parallel, a new computer system, developed for the management of course outlines and syllabuses and adapted to the implementation process, is in its pilot phase. At UQAM, the teaching of a number of courses of the engineering program, such as mathematics and natural sciences, is "outsourced" to non-engineering departments. It is therefore important to be able to rely on user-friendly tools to simplify processes and ensure the collaboration of everyone involved.

On a second front, an extensive bank of criteria for all the twelve attributes has been established, and a review of relevant indicators for each attribute has been performed. A list of key indicators for each attribute is being completed by the steering committee. These indicators will be instrumental in the development of assessment tools well adapted to the different types of attributes. Here again, much emphasis shall be put on the usability of the approaches selected.

Throughout the process, evaluation has quickly been identified as a key element, if not a potential irritant for instructors. Upcoming work sessions, which will notably be focusing on criterion-referenced assessment, should enable engineering professors to gradually become acquainted with tools for assessment of learning outcomes they are for now generally unfamiliar with. At further stages of the process, and once tools and indicators are sell established, other instructors (lecturers, instructors from other departments, etc.) will be asked to join the work groups.

The move towards criterion-referenced evaluation faces several challenges. Integration with the traditional grading scheme, which is mandatory as per university regulations, is not straightforward. Duplicating assessment or over-assessing, either by design or as a result of a lack of confidence in the new paradigm, is a classical pitfall that must be avoided.

\section{CONCLUSION}

The process of implementation is still in progress. Although UQAM offers only one engineering program, many other disciplines are engaged in similar processes in the Universite du Québec network. We hope that our partnership will lead to the creation of tools, procedures and mechanisms that can be transfered to other schools and faculties. 